\title{
Genome Data of Fusarium oxysporum f. sp. cubense Race 1 and Tropical Race 4 Isolates Using Long-Read Sequencing
}

Yingzi Yun, ${ }^{1}$ Aixia Song, ${ }^{1}$ JianDong Bao, ${ }^{1}$ Shasha Chen, ${ }^{1}$ Songmao Lu, ${ }^{1}$ Chunzhen Cheng, ${ }^{2}$ Wenhui Zheng, ${ }^{1}$ Zonghua Wang, ${ }^{1,3 \dagger}$ and Liangsheng Zhang ${ }^{1 \dagger}$

${ }^{1}$ State Key Laboratory of Ecological Pest Control for Fujian and Taiwan Crops, Fujian Provincial Key Laboratory of Haixia Applied Plant Systems Biology, Fujian Agriculture and Forestry University, Fuzhou, China

${ }^{2}$ College of Horticulture, Fujian Agriculture and Forestry University

${ }^{3}$ Institute of Oceanography, Minjiang University, Fuzhou, China

\begin{abstract}
Fusarium wilt of banana is caused by the soilborne fungal pathogen Fusarium oxysporum f. sp. cubense. We generated two chromosome-level assemblies of $F$. oxysporum f. sp. cubense race 1 and tropical race 4 strains using single-molecule real-time sequencing. The F. oxysporumf. sp. cubense race 1 and tropical race 4 assemblies had 35 and 29 contigs with contig N50 lengths of 2.08 and $4.28 \mathrm{Mb}$, respectively. These two new references genomes represent a greater than 100 -fold improvement over the contig N50 statistics of the previous short-read-based $F$. oxysporum f. sp. cubense assemblies. The two high-quality assemblies reported here will be a valuable resource for the comparative analysis of $F$. oxysporum $\mathrm{f}$. $\mathrm{sp}$. cubense races at the pathogenic level.
\end{abstract}

\section{Genome Announcement}

Fusarium oxysporum f. sp. cubense is the causal pathogen of Fusarium wilt of banana (Musa spp.) (O'Donnell et al. 1998; Siamak and Zheng 2018). Based on the infection host, F. oxysporum f. sp. cubense can be classified into four races. F. oxysporum f. sp. cubense race 1 (Foc1) nearly destroyed global banana export trade in the 19th century before the development of the Foc1-resistant banana cultivar Cavendish (Ploetz 2015). F. oxysporum f. sp. cubense tropical race 4 (FocTR4) is a new serious threat to banana cultivation due to its strong pathogenicity affecting nearly all banana cultivars, including Cavendish (Dita et al. 2018). Thus, improving the current understanding of the molecular mechanisms underlying pathogenicity and host specificity of $F$. oxysporum f. sp. cubense races is critical for developing sustainable control methods for this disease. In this regard, high-quality whole-genome sequence data of $F$. oxysporum f. sp. cubense strains are also needed to support this work.

Whole-genome sequencing technology and subsequent next-generation sequencing (NGS) strategies were used to generate genome assemblies for one Foc1 isolate (N2) and two Foc4 isolates (B2 and II5) (Guo et al. 2014). However, because these NGS assemblies are highly fragmented, it is difficult to perform in-depth analyses on genomic features that contribute to variation and pathogenesis among $F$. oxysporum f. sp. cubense races. To

Y. Yun and A. Song contributed equally to this work.

†Corresponding authors: L. Zhang; fafuzhang@163.com; and Z. Wang; zonghuaw@163.com

The author(s) declare no conflict of interest.

Accepted for publication 6 May 2019.

(C) 2019 The American Phytopathological Society

\section{Funding}

This research was supported by Natural Science Foundation of China (31601583) and Fujian Agriculture and Forestry University Outstanding Youth Scientific Research Project (xjq201625 and xjq201702).

Keywords

genomics 
Table 1. Genome characteristics and predicted features of the assembled Fusarium oxysporum f. sp. cubense strains

\begin{tabular}{|c|c|c|c|c|c|}
\hline \multirow[b]{2}{*}{ Characteristics } & \multicolumn{5}{|c|}{ Race } \\
\hline & Foc1 & FocTR4 & Foc1 & Foc4 & FocTR4 \\
\hline Genome strain & 60 & 58 & N2 & B2 & 115 \\
\hline Genome size $(\mathrm{Mb})$ & 48.56 & 48.19 & 46.91 & 48.81 & 46.43 \\
\hline Contig (count) & 35 & 29 & 2,175 & 3,824 & 716 \\
\hline Contig N50 size (Mb) & 2.134 & 4.378 & 0.146 & 0.028 & 0.349 \\
\hline Max contig size (Mb) & 6.686 & 6.688 & 0.646 & 0.116 & 1.738 \\
\hline Average contig size (Mb) & 1.388 & 1.662 & 0.022 & 0.013 & 0.065 \\
\hline Genome BUSCO (\%) & 99.6 & 100 & 99.3 & 98.9 & 100 \\
\hline Transposable element (\%) & 5.72 & 8.63 & 4.34 & 4.00 & 5.22 \\
\hline Genes (count) & 15,865 & 15,519 & 15,438 & 14,506 & 16,634 \\
\hline Genes BUSCO (\%) & 95.2 & 96.9 & 93.1 & 84.8 & 100 \\
\hline
\end{tabular}

address this limitation, we used single-molecule real-time (SMRT) sequencing developed by Pacific Biosciences to generate near-complete genome assemblies for Foc1 isolate 60 and FocTR4 isolate 58 , which were previously identified by our group (Wu et al. in press). Sequencing was performed at the Beijing Novogene Bioinformatics Technology Co., Ltd. For the PacBio library, 20-kb inserts for each strain were constructed using the SMRTbell Template Prep Kit 1.0. One SMRTCell was used to sequence the DNA library with the PacBio Sequel system. Low-quality reads were filtered by SMRT Link (v5.0.1) with default parameters (Pacific Biosciences). Finally, we obtained $2.86 \mathrm{~Gb}$ of long-read data (polymerase reads) for Foc1 strain 60, with an N50 and average length of 13,007 and 9,418 bp, respectively. For FocTR4 strain $58,6.58 \mathrm{~Gb}$ of long reads were collected, with an N50 and average length of 10,549 and $7,406 \mathrm{bp}$, respectively.

Genome assembly was conducted using Canu v1.5 (Koren et al. 2017), followed by two rounds of polishing with Quiver (Chin et al. 2013).The Foc1 60 genome assembly was approximately $47.42 \mathrm{Mb}$ long and included 35 contigs $(\mathrm{N} 50=2.08 \mathrm{Mb}$ ) with an average length of $1.35 \mathrm{Mb}$ (Table 1). The genome size of FocTR4 58 was $47.06 \mathrm{Mb}$, which consisted of 29 contigs ( $\mathrm{N} 50=4.28 \mathrm{Mb}$ ) with an average length of $1.62 \mathrm{Mb}$ (Table 1). The contig N50 statistics for the Foc1 60 and FocTR4 58 genome assemblies were more than 100-fold greater than those of Foc1 N2 and Foc4 B2. The completeness of the Foc1 60 and FocTR4 58 assemblies was evaluated using BUSCO v3.0 (Simão et al. 2015). This analysis indicated that the assemblies were robust and complete, with 99.6 and $100 \%$ of the fungal genes, respectively, detected in our assembly (Table 1). We used ALLMAPS v0.8.4 to order and orient the Foc1 60 and FocTR4 58 contigs relative to the equally weighted linkage map of $F$. oxysporum f. sp. lycopersici (Ma et al. 2010; Tang et al. 2015). Next, the 35 and 29 contigs of Foc1 60 and FocTR4 58, respectively, were anchored and oriented onto 11 core chromosomes, and 4 lineage-specific chromosomes of $F$. oxysporum f. sp. lycopersici 4287 that are rich in transposons and genes related to pathogenicity. F. oxysporum f. sp. lycopersici Chr03, Chr06, Chr14, and Chr15 were not found in Foc1 60 or FocTR4 58, which is consistent with the reported findings for Foc1 N2 and Foc4 B2 (Guo et al. 2014).

Transposable elements (TEs) were identified by ab initio prediction. Approximately 1.98 and $3.30 \mathrm{Mb}$ of TEs (4.18 and $7.01 \%$ of the assemblies) were detected in the Foc1 60 and FocTR4 58 assemblies, respectively. Intact protein-coding genes were identified using a combination of homology-based, ab initio, and transcriptome-based prediction methods. A total of 15,865 and 15,519 intact protein-coding gene models were predicted as consensus gene sets in Foc1 60 and FocTR4 58, respectively (Table 1). Evaluation using BUSCO v3.0 showed that 95.2 and $96.9 \%$ of conserved BUSCO proteins (fungi_odb9) were present in Foc1 60 and FocTR4 58, respectively (Table 1). In total, we found that 95.36 and $96.01 \%$ of genes in Foc1 60 and FocTR4 58, respectively, could be annotated with InterProScan, Pfam, Gene Ontology, Kyoto Encyclopedia of Genes and Genomes, and NR (from NCBI). The DNA sequence data, genome assemblies, and annotation for genes and TEs have been deposited into BIG Sub under accession number PRJCA001282.

Author-Recommended Internet Resource

BIG Sub: http://bigd.big.ac.cn/bioproject/browse/PRJCA001282 


\section{Literature Cited}

Chin, C. S., Alexander, D. H., Marks, P., Klammer, A. A., Drake, J., Heiner, C., Clum, A., Copeland, A., Huddleston, J., Eichler, E. E., Turner, S. W., and Korlach, J. 2013. Nonhybrid, finished microbial genome assemblies from long-read SMRT sequencing data. Nat. Methods 10:563-569.

Dita, M., Barquero, M., Heck, D., Mizubuti, E. S. G., and Staver, C. P. 2018. Fusarium wilt of banana: Current knowledge on epidemiology and research needs toward sustainable disease management. Front. Plant Sci. 9:1468.

Guo, L., Han, L., Yang, L., Zeng, H., Fan, D., Zhu, Y., Feng, Y., Wang, G., Peng, C., Jiang, X., Zhou, D., Ni, P., Liang, C., Liu, L., Wang, J., Mao, C., Fang, X., Peng, M., and Huang, J. 2014. Genome and transcriptome analysis of the fungal pathogen Fusarium oxysporum f. sp. cubense causing banana vascular wilt disease. PLoS One 9:e95543.

Koren, S., Walenz, B. P., Berlin, K., Miller, J. R., Bergman, N. H., and Phillippy, A. M. 2017. Canu: Scalable and accurate long-read assembly via adaptive $k$-mer weighting and repeat separation. Genome Res. 27:722-736.

Ma, L. J., van der Does, H. C., Borkovich, K. A., Coleman, J. J., Daboussi, M. J., Di Pietro, A., Dufresne, M., Freitag, M., Grabherr, M., Henrissat, B., Houterman, P. M., Kang, S., Shim, W. B., Woloshuk, C., Xie, X., Xu, J. R., Antoniw, J., Baker, S. E., Bluhm, B. H., Breakspear, A., Brown, D. W., Butchko, R. A. E., Chapman, S., Coulson, R., Coutinho, P. M., Danchin, E. G. J., Diener, A., Gale, L. R., Gardiner, D. M., Goff, S., Hammond-Kosack, K. E., Hilburn, K., Hua-Van, A., Jonkers, W., Kazan, K., Kodira, C. D., Koehrsen, M., Kumar, L., Lee, Y. H., Li, L., Manners,
J. M., Miranda-Saavedra, D., Mukherjee, M., Park, G., Park, J., Park, S. Y., Proctor, R. H., Regev, A., Ruiz-Roldan, M. C., Sain, D., Sakthikumar, S., Sykes, S., Schwartz, D. C., Turgeon, B. G., Wapinski, I., Yoder, O., Young, S., Zeng, Q., Zhou, S., Galagan, J., Cuomo, C. A., Kistler, H. C., and Rep, M. 2010. Comparative genomics reveals mobile pathogenicity chromosomes in Fusarium. Nature 464:367-373.

O'Donnell, K., Kistler, H. C., Cigelnik, E., and Ploetz, R. C. 1998. Multiple evolutionary origins of the fungus causing Panama disease of banana: Concordant evidence from nuclear and mitochondrial gene genealogies. Proc. Natl. Acad. Sci. U.S.A. 95:2044-2049.

Ploetz, R. C. 2015. Fusarium wilt of banana. Phytopathology 105:1512-1521.

Siamak, S. B., and Zheng, S. J. 2018. Banana Fusarium wilt (Fusarium oxysporum f. sp. cubense) control and resistance, in the context of developing wilt-resistant bananas within sustainable production systems. Hortic Plant J 4:208-218.

Simão, F. A., Waterhouse, R. M., loannidis, P., Kriventseva, E. V., and Zdobnov, E. M. 2015. BUSCO: Assessing genome assembly and annotation completeness with single-copy orthologs. Bioinformatics 31:3210-3212.

Tang, H., Zhang, X., Miao, C., Zhang, J., Ming, R., Schnable, J. C., Schnable, P. S., Lyons, E., and Lu, J. 2015. ALLMAPS: Robust scaffold ordering based on multiple maps. Genome Biol. 16:3.

Wu, K.-L., Chen, W.-Z., Yang, S., Wen, Y., Zheng, Y.-R., Anjago, W. M., Yun, Y.-Z., and Wang, Z.-H. Isolation and identification of Fusarium oxysporum f. sp. cubense in Fujian Province, China. J. Integr. Agric. In press. 